\title{
Spin effects in bottomonium at Belle
}

\author{
Umberto Tamponi* \\ INFN - Sezione di Torino \\ Universita' di Torino \\ E-mail: tamponiato.infn.it
}

\begin{abstract}
Spin effects have a dominant role in the understanding of the dynamic of the Bottomonium system, both in the transitions and in the correlation among light hadrons being produced in their annihilation. Naively we expect a suppression of the transitions with spin flipping terms, but recent results on $\eta$ transitions from $\Upsilon(4 S)$ and $\Upsilon(5 S)$ challenge this approach. The most recent results from Belle on this field will help to clarify the role of the spin in the modeling of the singleand two-meson transitions. Besides this the Belle collaboration is performing an extensive series of studies on baryon production in bottomonium decays, meant to address the two-baryon correlation in $\Upsilon(1 S)$ annihilation and the precise measurement of hyperon inclusive production rates in $e^{+} e^{-}$collision. The search for the long sought $H$ dibaryon is part of this effort.
\end{abstract}

XXII. International Workshop on Deep-Inelastic Scattering and Related Subjects, 28 April - 2 May 2014

Warsaw, Poland

* Speaker. 


\section{Bottomonium transitions and spectroscopy}

In the past lustrum quarkonium physics has seen an amazing outbreak of results [1]. In particular, the elusive spin singlet states, the $h_{b}(n P)$ and $\eta_{b}(n S)$, have been finally observed in a number of different processes. Historically the access to these states was thought to be possible only trough the suppressed radiative transition $\Upsilon(n S) \rightarrow \gamma \eta_{b}(m S)$ [2, 3, 4], and the hadronic, isospin suppressed transition $\Upsilon(3 S) \rightarrow \pi^{0} h_{b}(1 P)$ [5]. Only recently it has been possible to provide a completely independent measurement of the $\eta_{b}(1 S)$ parameters and the first measurement of the $\eta_{b}(2 S)$ ones, thanks to the dipion transitions $\Upsilon(5 S) \rightarrow \pi^{+} \pi^{-} h_{b}(n P)$ [6] and the subsequent radiative decay $h_{b}(n P) \rightarrow \gamma \eta_{b}(m S)$ [7]. A precise measurement of the $\eta_{b}(n S)$ masses and the corresponding hyperfine splittings provides a crucial the test of the spin-dependent part of the $b \bar{b}$ potential. The hyperfine splittings in the quarkonium are defied as the mass difference between the spin singlet and corresponding spin triplet states. The spin term of the $b-\bar{b}$ potential is proportional to $\delta(r)$ [8], where $r$ is the distance between the two quarks, thus the hyperfine splitting is proportional to the radial wavefunction evaluated in the origin, $\Delta M_{H F} \propto\left|\psi_{r}(0)\right|^{2}$. The hyperfine splitting of the $\mathrm{P}$-wave system is thus predicted to be 0 in every model, while in the $\mathrm{S}$-wave different models give different predictions. The results of the first measurements of the $\eta_{b}(1 P)$ mass show a striking discrepancy with the potential non relativistic QCD (pNRQCD) prediction [9] and tension with the lattice calculation [10]. The value of the $\eta_{b}(1 S)$ mass measured in the hadronic transitions from the $\Upsilon(5 S)$ is, on the contrary, in agreement with both the theoretical predictions. The transition $\Upsilon(5 S) \rightarrow \pi^{+} \pi^{-} h_{b}(n P)$ itself, not expected in the most popular theoretical models, has been subject of intense studies that allowed to observe, for the first time, the $h_{b}(2 P)$, the $\eta_{b}(2 S)$ and the, quarkonium-like states $Z_{b}$ and $Z-b^{\prime}[11,12,13]$.

The observation of the $\Upsilon(5 S) \rightarrow \pi^{+} \pi^{-} h_{b}(1 P)$ is part of series of studies on the single and double meson transition, whose dynamic is expected to be largely determined by the heavy quark spin properties. The NonRelativistic QCD (NRQCD) is one of the most common framework used in making prediction on the branching fraction of the transitions among bottomonium states. In the QCD Multipole Expansion (QCDME) approach, in particular, the QCD interaction Hamiltonian can be expanded in a series of multipoles, reminding the usual electromagnetic multipole series expansion [16]. In this

Table 1: Ratio $\frac{\mathscr{B}[\Upsilon(n S) \rightarrow \eta \Upsilon(m S)]}{\mathscr{B}[\Upsilon(n S) \rightarrow \pi \pi \Upsilon(m S)]}$ measured by the experiment, compared with the value predicted within the QCDME model. The $\eta$ transitions require the heavy quark spin flip and are thus suppressed with respect to the $\pi \pi$ ones. The experimental values are derived from [14] and [15].

\begin{tabular}{lcc}
\hline transition & Experiment & QCDME \\
\hline$\Upsilon(2 S) \rightarrow \Upsilon(1 S)$ & $2 \times 10^{-3}$ & $\approx 2 \times 10^{-3}$ \\
$\Upsilon(3 S) \rightarrow \Upsilon(1 S)$ & $<2 \times 10^{-3}$ & $\approx 6 \times 10^{-3}$ \\
$\Upsilon(4 S) \rightarrow \Upsilon(1 S)$ & 2.4 & $<<1$ \\
$\Upsilon(5 S) \rightarrow \Upsilon(1 S)$ & 0.1 & $<<1$ \\
$\Upsilon(5 S) \rightarrow \Upsilon(2 S)$ & 0.4 & $<<1$ \\
\hline \hline
\end{tabular}
formalism the presence of a chromomagnetic amplitude, that is responsible for the spin flip of the heavy quark, suppresses the transition by a factor proportional to $m_{b}^{-2}$. Particularly interesting is the comparison between the $\Upsilon(n S) \rightarrow \eta \Upsilon(m S)$ transitions, which are spin flipping, and the corresponding $\pi^{+} \pi^{-}$ones that do no require the $b$ quark spin to flip. Table ?? reports the ratio ratio $\frac{\mathscr{B}[\Upsilon(n S) \rightarrow \eta \Upsilon(m S)]}{\mathscr{B}[\Upsilon(n S) \rightarrow \eta \Upsilon(m S)]}$. Strong discrepancies can be 
observed in the $\Upsilon(4 S)$ and $\Upsilon(5 S)$ cases. The $\Upsilon(2 S) \rightarrow \eta \Upsilon(1 S)$ transition has been measured by CLEO [17], Babar [18] and Belle [19]. The result reported by Belle is consistent with the theory prediction in Ref. [14], even if most theory predictions foresee a larger widths for this transition [15]. At the same time, for the $\Upsilon(3 S) \rightarrow \eta \Upsilon(1 S)$ transition, the best upper limit [18] is already a factor two below the theory expectations. The observation of a surprisingly large ratio from $\Upsilon(4 S)$ was not foreseen by any theory paper [20]. After this observation, a number of theoretical works have done predictions on the $\eta$ transitions from higher bottomonia. On the experimental side, Belle observed large $\eta$ transitions from $\Upsilon(5 S)$ to $\Upsilon(1,2 S)$ in exclusive mode. With the increasing radius of the decaying state, the QCDME based-predictions become no more reliable, due to the presence of intermediate states like the $Z_{b}$ and $Z_{b}^{\prime}$ or, possibly, due to coupled channel effects. In both cases, we observe that the heavy quark spin symmetry is no more the dominant effect in the states above the open beauty threshold, where the dynamic of the light quark content of the quarkonium states becomes more important.

\subsection{Progresses on the $\eta$ transitions}

Even if the experimental pattern is almost complete in the sector of the $\Upsilon(n S) \rightarrow$ $\eta \Upsilon(m S)$ hadronic transition, no experimental result is available on the $\eta$ transitions among spin triplet and spin singlet states. The $\Upsilon(4 S) \rightarrow \eta h_{b}(1 P)$ in particular is expected to have a branching fraction $\mathscr{B}\left[\Upsilon(4 S) \rightarrow \eta h_{b}(1 P)\right] \approx 10^{-3}$ [21]. For the $\Upsilon(5 S)$ no theoretical predictions are available. We present here the preliminary results on the $\eta$ transitions from $\Upsilon(4 S)$ and $\Upsilon(5 S)$ to the $h_{b}(n P)$ obtained using the data collected by the Belle detector $[22,23]$ at the KEKB $e^{+} e^{-}$collider [24]. In addition, the radiative transition $h_{b}(n P) \rightarrow \gamma \eta_{b}(m S)$ can be used in order to measure the resonance parameters of the $\eta_{b}(m S)$. Since no $h_{b}(1,2 P)$ exclusive decay modes are known to have a significant branching fraction and a clear experimental signature, the analysis is performed inclusively: The signals of $\Upsilon(4,5 S) \rightarrow \eta(b \bar{b})$

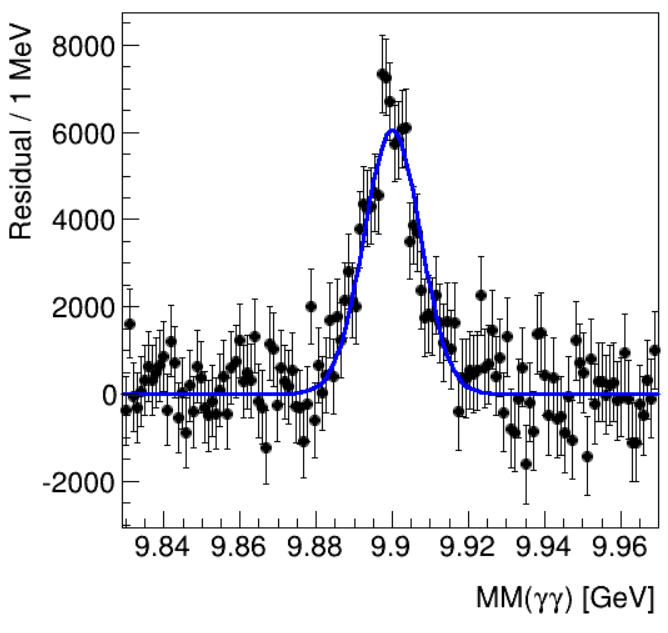

Figure 1: The $\eta$ recoil mass spectrum in the $\Upsilon(4 S)$ dataset, after the subtraction of the combinatorial background in the $h_{b}(1 P)$ region. The blue, solid line represents the best fit, obtained with the sum of a 6 - th order polynomial and a gaussian peak. transitions are expected to appear as peaks in the recoil mass spectrum of the $\eta$ candidates in the samples of hadronic events collected at the $\Upsilon(4,5 S)$ energies. We compute the missing mass $M_{\text {miss }}$ of the $\gamma \gamma$ pairs with invariant mass within $0.48 \mathrm{GeV} / \mathrm{c}^{2}$ and $0.62 \mathrm{GeV} / \mathrm{c}^{2}$, after applying a kinematic fit with mass constraint. The obtained distribution is dominated by a large combinatorial background, that can be described by a polynomial Probability Density Function (PDF). Figure 1 shows the residual from the background PDF in the $h_{b}(1 P)$ region, in the $\Upsilon(4 S)$ dataset. The 
$\Upsilon(4 S) \rightarrow \eta h_{b}(1 P)$ peak is observed for the first time, with a statistical significance greater than $5 \sigma$, and the corresponding branching ratio is $\mathscr{B}\left[\Upsilon(4 S) \rightarrow \eta h_{b}(1 P)\right]=(1.83 \pm 0.16 \pm 0.17) \times 10^{-3}$.

The same analysis performed at the $\Upsilon(5 S)$ energy shows significant peaks in correspondence of the $\Upsilon(5 S) \rightarrow \eta \Upsilon(2 S)$, and $\Upsilon(5 S) \rightarrow \eta \Upsilon(1 D)$, with statistical significance of $3.1 \sigma$ and $4.4 \sigma$, respectively. (Figure 2).

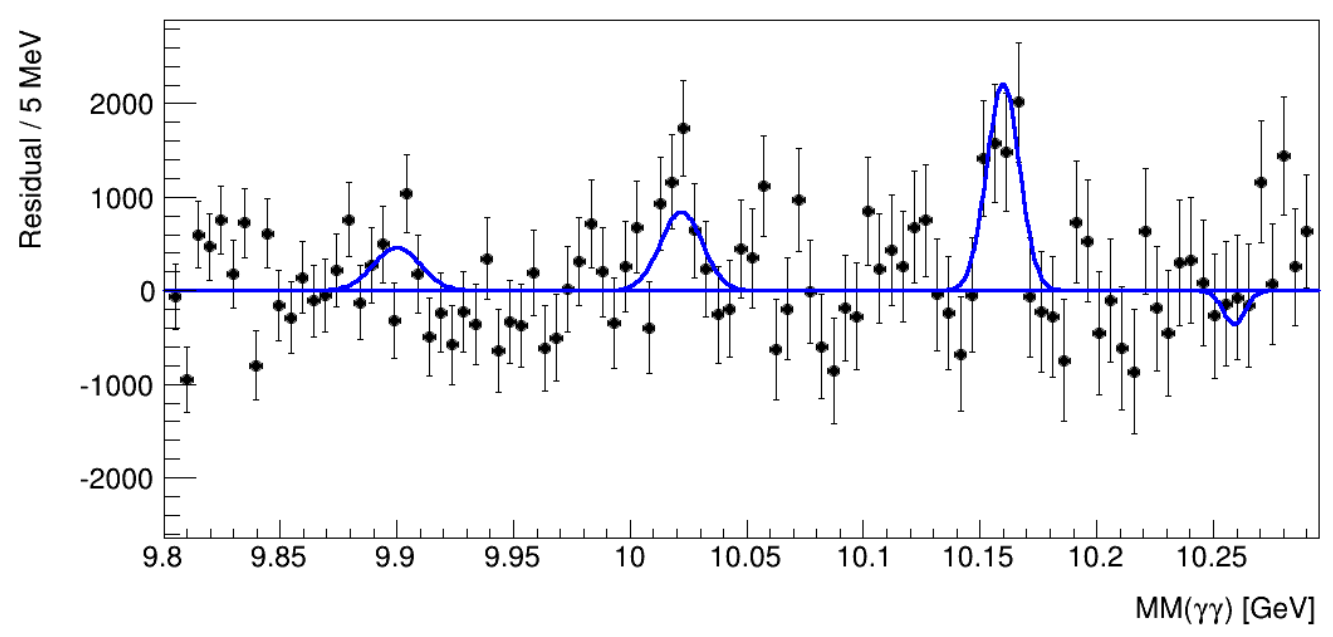

Figure 2: The $\eta$ recoil mass spectrum in the $\Upsilon(5 S)$ dataset, after the subtraction of the combinatorial background. The blue, solid line represents the best fit, obtained with the sum of a $6-t h$ order polynomial and four gaussian peaks.

A summary of the measured branching ratios is presented in Table 2. These results allow for the first time to measure the ratios $\frac{\mathscr{B}\left[\Upsilon(n S) \rightarrow \eta h_{b}(m P)\right]}{\mathscr{B}\left[\Upsilon(n S) \rightarrow \pi \pi h_{b}(m P)\right]}$. In the $\Upsilon(4 S)$ decay a large violation of the predictions based on the heavy quark spin symmetry were observed in the transitions to the $\Upsilon(1 S)$. The lower limit we obtain in the case of the $h_{b}(1 P)$ transitions, $\frac{\mathscr{B}\left[\mathrm{Y}(n S) \rightarrow \eta h_{b}(m P)\right]}{\mathscr{B}\left[\mathrm{Y}(n S) \rightarrow \pi \pi h_{b}(m P)\right]}>2.0(90 \% \mathrm{CL})$, is in reasonable agreement with the QCDME expectations. The interpretation of the $\Upsilon(5 S)$ results is not straightforward, since the $\Upsilon(5 S) \rightarrow \pi^{+} \pi^{-} h_{b}(n P)$ proceed almost en-

Table 2: Summary of the results of the searches for $\Upsilon(n S) \rightarrow \eta(b \bar{b})$. The branching ratio $\mathscr{B}$ is reported. If the statistical significance is lower than $3 \sigma$, the $90 \%$ CL upper limit on $\mathscr{B}$ is reported.

\begin{tabular}{lc}
\hline Process & $\mathscr{B}$ \\
\hline$\Upsilon(5 S) \rightarrow \eta \Upsilon(2 S)$ & $(2.1 \pm 0.7 \pm 0.3) \times 10^{-3}$ \\
$\Upsilon(5 S) \rightarrow \eta \Upsilon(1 D)$ & $(2.8 \pm 0.7 \pm 0.4) \times 10^{-3}$ \\
$\Upsilon(5 S) \rightarrow \eta h_{b}(1 P)$ & $<3.3 \times 10^{-3}$ \\
$\Upsilon(5 S) \rightarrow \eta h_{b}(2 P)$ & $<3.7 \times 10^{-3}$ \\
$\Upsilon(4 S) \rightarrow \eta h_{b}(1 P)$ & $(1.83 \pm 0.16 \pm 0.17) \times 10^{-3}$ \\
\hline
\end{tabular}
tirely trough the $Z_{b}$ 's.

\subsection{Measurement of the $\eta_{b}(1 S)$ resonance parameters}


We present a new, preliminary measurement of the mass of the $\eta_{b}(1 S)$, obtained using the process $\Upsilon(4 S) \rightarrow \eta h_{b}(1 P) \rightarrow$ $\eta \gamma \eta_{b}(1 S)$. We measure the missing mass difference $\Delta M M=M M(\eta \gamma)-M M(\eta)$, where $M M(\eta)$ is the missing mass from the $\eta$ candidate and $M M(\eta \gamma)$ is the missing mass of the $\eta \gamma$ system. By fitting the $\Upsilon(4 S) \rightarrow \eta h_{b}(1 P)$ yield as function of $\triangle M M$, we obtain the distribution shown in Figure 3. We measure $M_{\eta_{b}(1 S)}=9405.3 \pm 1.3 \pm 3.0$ $\mathrm{MeV} / \mathrm{c}^{2}$, corresponding to an hyperfine splitting $\Delta M_{H F}(1 S)=55.0 \pm 1.3 \pm 3.2 \mathrm{MeV} / \mathrm{c}^{2}$, in agreement with the lattice and pNRQCD predictions $[9,10]$.

\section{Low energy effects in $\Upsilon(n S)$ decays}

The decay in open flavor is forbidden for $\Upsilon(1 S), \Upsilon(2 S)$ and $\Upsilon(3 S)$ since their are

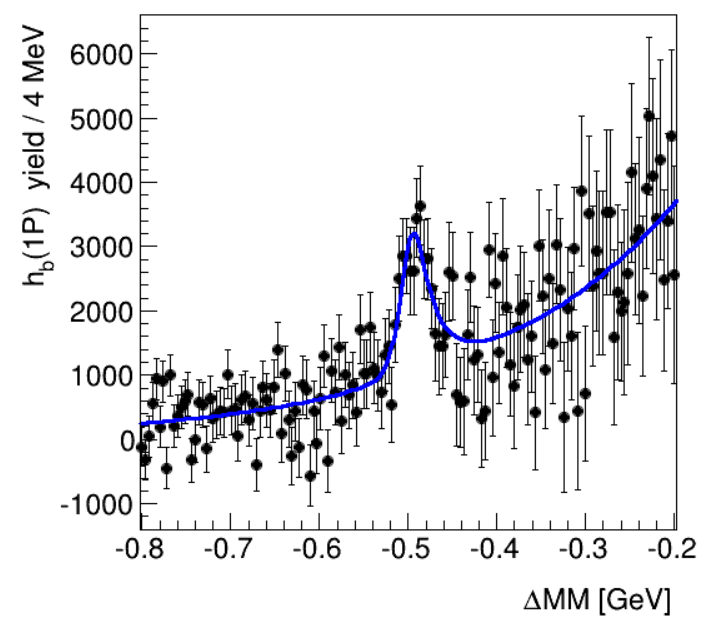

Figure 3: $\Upsilon(4 S) \rightarrow \eta h_{b}(1 P)$ yield as function of $\Delta M M$. The peak at $\Delta M M \approx-0.5 \mathrm{GeV} / \mathrm{c}^{2}$ is due to the $h_{b}(1 P) \rightarrow \gamma \eta_{b}(1 S)$ transition. The blue solid line represents our best fit. located below the $B \bar{B}$ threshold, thus these states primarily annihilate in three gluons. Since the typical radius of the $\Upsilon$ states is of the order of $0.15-0.3 \mathrm{fm}[1]$, the $\Upsilon(n S)$ annihilation provides a state with very high initial partonic density. In this situation the formation of multy quark states or bound states of known hadrons is expected to be favoured. Indeed, a significant production of anti-deuteron in $\Upsilon(1 S), \Upsilon(2 S)$ and $\Upsilon(3 S)$ annihilation [25] has been reported. In the early years of bottomonium physics, the ARGUS collaboration studied in details the inclusive production of hyperons' that arise from the gluon fragmentation, finding a sizable discrepancy between the data and the MonteCarlo-based predictions [26]. Twenty years later the CLEO collaboration confirmed this discrepancy in the $\Lambda$ and $\phi$ meson production [27], suggesting a general mismodelling the strange quark hadronization mechanism in the current MonteCarlo models. The first studies on $\Upsilon(n S)$ exclusive annihilation modes became available only recently [28, 29, 30], and none of them address the problem of hyperons' production, and the mechanisms that are at the origin of the high inclusive yield of strange baryons remain largely un-investigated. A preliminary study of the exclusive production modes of $\Lambda$ has been done by the Belle collaboration: $\Upsilon(1 S), \Upsilon(2 S)$ and $\chi_{b J}(1 P)$ annihilations with a $\Lambda \bar{\Lambda}$ pair, up to three additional pairs of light mesons $\pi^{+} \pi^{-}, K^{+} K^{+}$and $p \bar{p}$ and up to one $\pi^{0}$, for a total of 48 independent decay channels, were investigated. Signals with statistical significance greater than $5 \sigma$ are observed in eleven $\Upsilon(1 S)$ and three $\Upsilon(2 S)$ decay modes. The study of the $\Lambda \bar{\Lambda}$ invariant mass in the reconstructed events shows, in some cases, a strong deviation from the phase-space flat distribution. The deviation consists in an accumulation in the near-threshold region. This is the first observation of such a behavior in bottomonium decays. Even if no theoretical work addressed the near threshold enhancement in the three gluon annihi- 
lations, this is a well known and still debated phenomenon in the $B$ meson sector [?] and in the charmonium radiative decays $[35,36]$. The sum of the observed decays only account for less than $1 \%$ of the total number of hadronic annihilations, suggesting that the $\Lambda$ production mainly occurs in high multiplicity events, where the average momentum per track is lower. This result suggests that the hyperons came mainly from the soft and ultra-soft partons' fragmentation. The high yield of $\Lambda$ suggest the possibility of search for one of the most famous exotic states, the $H$-dibaryon postulated by Jaffe in 1977 [37] as a fully antisymmetry arrangement of six quarks $u d s u d s$, in quarkonium annihilation; a deuteron-like $\mathrm{H}$ dibaryon, with mass close to the $\Lambda \Lambda$ thresholds could be copiously produced. According to its mass, the $\mathrm{H}$ can decay in different modes: if $m_{H}>2 m_{\Lambda}$ the $\Delta S=0$ decay $H \rightarrow \Lambda \Lambda$ is expected to be dominant, replaced, if the mass is sufficient, by $H \rightarrow \Xi^{-} p$; below the $\Lambda \Lambda$ threshold $\Delta S=0$ transitions are forbidden by the conservation of the energy and the baryonic number, thus only the $\Delta S=1$ modes $\Lambda p \pi^{-}, \Lambda n, \Sigma^{0} n$ or $\Sigma^{+} \pi^{-}$are allowed.

The Belle collaboration searched for $H \rightarrow \Lambda \Lambda, H \rightarrow \Lambda p \pi^{-}[38]$ and $H \rightarrow \Xi^{-} p$ (preliminary) modes in the region close to $2 M_{\Lambda}$, without finding any peak in any invariant mass distribution. Assuming the intrinsic width of the $H$ to be negligible, a $90 \%$ confidence level mass dependent upper limit has been derived; this measurement strongly disfavor the presence of a $\mathrm{H}$ dibaryon with dynamic similar to the deuteron, since on average $\mathscr{B}[\Upsilon(1,2 S) \rightarrow$ $H+X]<20 \times[\Upsilon(1,2 S) \rightarrow H+X]$ (Fig. 4). Nevertheless the measurement does not completely rule out the presence of the $\mathrm{H}$

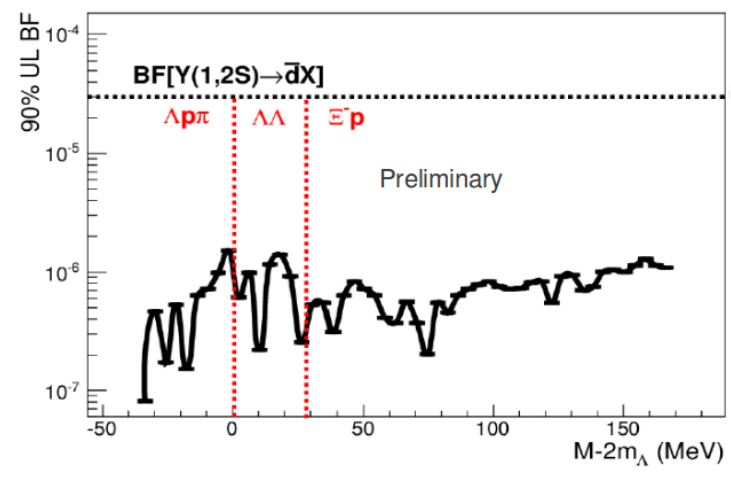

Figure 4: Upper limit for the production of $H$ dibaryon from in $\Upsilon(1 S)$ and $\Upsilon(2 S)$ annihilations, in its decay modes $H \rightarrow \Lambda \Lambda, H \rightarrow$ $\Lambda p \pi^{-}$and $H \rightarrow \Xi^{-} p$. dibaryon: the production mechanism of the $\bar{d}$ has not been studied in detail with a precision measurement of the spectrum characteristics, but the most common theoretical model for its description is based on the recombination of $\bar{p}$ and $\bar{n}$ after the chemical freeze out [39], thus if the $\mathrm{H}$ is a bound state of six quark and not a deuteron-like state, the production mechanism could be radically different and the branching fraction cannot be directly compared.

\section{References}

[1] N. Brambilla et al., Eur. Phys. J. C 71 (2011) 1534.

[2] B. Aubert et al. [BaBar Collaboration], Phys. Rev. Lett. 103 (2009) 161801.

[3] G. Bonvicini et al. [CLEO Collaboration], Phys. Rev. D 81 (2010) 031104.

[4] S. Sandilya et al. [Belle Collaboration], Phys. Rev. Lett. 111 (2013) 112001.

[5] J.P. Lees et al. (BaBar Collaboration), Phys. Rev. D 84, 112002(R) (2008).

[6] I. Adachi et al. [Belle Collaboration], Phys. Rev. Lett. 108 (2012) 032001. 
[7] R. Mizuk et al. [Belle Collaboration], Phys. Rev. Lett. 109 (2012) 232002.

[8] M. Werner, Search for new bottomonium(-like) states in $e^{+} e \rightarrow B \bar{B} \pi \pi$ at the BELLE experiment Master Thesis, Justus-Leabig Universitat Giessen (2010).

[9] B. A. Kniehl et al. , Phys. Rev. Lett. 92 (2004) 242001 [Erratum-ibid. 104 (2010) 199901].

[10] S. Meinel, Phys. Rev. D 82 (2010) 114502.

[11] A. Bondar et al. [Belle Collaboration], Phys. Rev. Lett. 108 (2012) 122001.

[12] I. Adachi et al. (Belle Collaboration), Phys. Rev. Lett. 108, 032001 (2012).

[13] P. Krokovny et al. (Belle Collaboration), Phys. Rev. D 88, 052016 (2013).

[14] M. B. Voloshin, Prog. Part. Nucl. Phys. 61, 455 (2008).

[15] Y. .A. Simonov and A. I. Veselov, Phys. Lett. B 673, 211 (2009).

[16] Y. -P. Kuang, Front. Phys. China 1, 19 (2006).

[17] Q. He et al. (CLEO Collaboration), Phys. Rev. Lett. 101, 192001 (2008).

[18] J. P. Lees et al. (BaBar Collaboration), Phys. Rev. D 84, 092003 (2011).

[19] U. Tamponi et al. (Belle Collaboration), Phys. Rev. D 87, 011104 (2013).

[20] B. Aubert et al. (BaBar Collaboration), Phys. Rev. D 78, 112002 (2008)

[21] F. -K. Guo, C. Hanhart and U. -G. Meissner, Phys. Rev. Lett. 105, 162001 (2010).

[22] A. Abashian et al., Nucl. Instrum. Meth. A 479, 117 (2002).

[23] J. Brodzicka et al. [for the Belle Collaboration], Prog. Theor. Exp. Phys. 04D001 (2012).

[24] S. Kurokawa, E. Kikutani, Nucl. Instrum. Meth. A 499, 1 (2003), and other papers included in this volume.

[25] D.M. Asner et al. [CLEO Collaboration], Phys. Rev. D75, 012009 (2007).

[26] H. Albrecht et al. [ARGUS Collaboration], Z. Phys. C 39, 177 (1988).

[27] R.A. Briere et al. [CLEO collaboration], Phys. Rev. D76, 012005 (2007).

[28] C.P. Shen et al. (Belle collaboration), Phys. Rev. D86, 031102 (2012).

[29] C.P. Shen et al. (Belle collaboration), Phys. Rev. D88, 011102(R) (2013).

[30] S. Dobbs et al., Phys. Rev. D86, 052003(R) (2013).

[31] X. Fu et al. (CLEO Collaboration), Phys. Rev. Lett. 79, 3125 (1997).

[32] K. Abe et al. (Belle Collaboration), Phys. Rev. Lett. 88, 181803 (2002).

[33] M. Suzuki, J. Phys. G34, 283 (2007).

[34] M.-Z. Wang et al. (Belle Collaboration), Phys. Lett. B 617, 141âĂŞ149 (2005)

[35] J.Z. Bai, et al. (BES Collaboration), Phys. Rev. Lett. 86, 022001 (2003).

[36] M. Ablikim, et al. (BES Collaboration), Phys. Rev. Lett. 95, 262001 (2005).

[37] R. L. Jaffe, Phys. Rev. Lett. 38 (1977) 195 [Erratum-ibid. 38 (1977) 617].

[38] B. H. Kim et al. [Belle Collaboration], Phys. Rev. Lett. 110 (2013) 222002.

[39] A. Ibarra and S. Wild, JCAP 1302 (2013) 021. 